\title{
Internal Electric Fields of Flexible GaAs Solar Cells Fabricated Using Epitaxial Lift-off
}

\author{
Received November 20, 2019; revised December 6, 2019; accepted December 9, 2019
}

\author{
Mo Geun So ${ }^{a, \#}$, Hyun Jun Jo ${ }^{b, \#, ~ G y o u n g ~ D u ~ P a r k ~}{ }^{b}$, Jae-Jin Shimc, Woo Kyoung Kimc, Yeongho Kim ${ }^{d}$, Sang Jun Lee ${ }^{d}$, and \\ Jong Su Kim ${ }^{\mathrm{b}, *}$ \\ ${ }^{a}$ Advanced Photonics Research Institute, Gwangju Institute of Science and Technology, Gwangju 61005, Republic of Korea

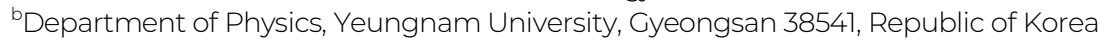 \\ 'School of Chemical Engineering, Yeungnam University, Gyeongsan 38541, Republic of Korea \\ ${ }^{d}$ Korea Research Institute of Standards and Science, Daejeon 34113, Republic of Korea \\ ${ }^{\#}$ Contributed equally to this work as first author.
}

*Corresponding author E-mail: jongsukim@ynu.ac.kr

\begin{abstract}
We have investigated the optical and electrical properties of flexible GaAs solar cells (SCs) fabricated by the epitaxial lift-off process using photoreflectance (PR) spectroscopy. The as-grown GaAs solar cells were transferred on Au/polyimide (ELA) and polydimethylsiloxane (PDMS; ELP) flexible substrates. In the PR spectra, low energy interference oscillations by internal multi-reflection were observed below the GaAs transition signals. The internal electric fields $\left(\boldsymbol{F}_{\text {int }}\right)$ were calculated to be 98.7 and $81.8 \mathrm{kV} / \mathrm{cm}$ for the ELA and ELP SCs, respectively. SCs produced by the ELA process exhibited fewer defects and higher $\boldsymbol{F}_{\text {int }}$ than the ELP SC.
\end{abstract}

Keywords: GaAs, Solar cell, Epitaxial lift-off, Defects

\section{Introduction}

GaAs is a direct transition semiconductor with high light absorption characteristics and theoretically has a band gap energy $(1.42 \mathrm{eV})$ near $1.40 \mathrm{eV}$ that can maximize the efficiency of solar cells (SCs). The efficiencies of GaAs SCs have been reported to be up to $29.1 \%$ in single junction structures [1]. In order to increase the efficiency of SCs by increasing the short-circuit current $\left(\mathrm{J}_{\mathrm{SC}}\right)$, methods of extending the absorption wavelength range or increasing the number of photogenerating carriers have been used. For this purpose, approaches such as preparing surface anti-reflective coatings using nanostructures, controlling the thickness of the absorption layer using light-trapping, and introducing quantum dots into the active layer have been used [2-5]. In addition, multi-junction SCs have been introduced to increase the open-circuit voltage $\left(\mathrm{V}_{\mathrm{OC}}\right)$. One of the fabrication methods for the multi-junction structure is wafer bonding using the epitaxial lift-off (ELO) method [6]. The ELO technology is also widely used in flexible SCs for fabricating lightweight and wearable devices [6-10]. A general ELO method is to separate an epilayer and a substrate by wet-etching a sample on which thin film growth is completed, and transfer or bond the separated epilayer to a desired substrate $[10,11]$. The SC devices fabricated through the ELO method have the advantage of reducing the cost of SC fabrication because the substrate can be reused.

High quality GaAs SCs fabricated through ELO using an AlAs sacrificial layer and wet-etching with HF solution have been reported $[11,12]$. Many studies have investigated how the device characteristics are affected by the temperature and concentration of the etching solution in the wet-etching process $[8,9]$. However, research on the effects of defects generated during the ELO process on the efficiency and internal electric field of SCs is still insufficient.

In this study, the optical properties of a sample transferred to a polydimethylsiloxane (PDMS) substrate using ELO-processing (ELOlayer/PDMS; ELP) and a sample bonded to an Au/polyimide layer (ELO-layer/Au/polyimide; ELA) were investigated using excitation intensity $\left(\mathrm{I}_{\mathrm{ex}}\right)$ and temperature-dependent photoreflectance (PR). The defects generated during the ELO process were studied by comparing the photoluminescence (PL) measurement results for ELP and ELA.

\section{Experimental methods}

The $\mathrm{n}^{+}-\mathrm{n}-\mathrm{p}^{+}$GaAs SCs were grown on a GaAs substrate using molecular beam epitaxy for the ELO process. In order to proceed with the ELO process, the GaAs (150 nm)/AlAs $(15 \mathrm{~nm}) / \mathrm{GaAs}(150 \mathrm{~nm}) /$ InGaP $(50 \mathrm{~nm})$ structures were grown on the $\mathrm{n}^{+}$-GaAs substrate. At this time, the AlAs sacrificial layer was used as an etching layer to separate the epilayer and substrate through wet-etching. The InGaP layer was used as an etch stop layer through a chemical mechanical polishing (CMP) process. Figures 1(a) and 1(b) show the ELA and ELP SC structures after the ELO process. After the ELO process, the ELA and ELP SCs exhibited $\mathrm{p}^{+}-\mathrm{n}-\mathrm{n}^{+}$structures. The reference GaAs (Ref) SC was grown on a GaAs substrate; the resultant SC exhibited a $\mathrm{p}^{+}-\mathrm{n}-\mathrm{n}^{+}$structure. In the ELA sample, $\mathrm{Au}$ was deposited on the polyimide thin film and sample on which growth was completed, and then Au-to-Au bonding was performed. The AlAs sacrificial layer was 
(a) ELA

\begin{tabular}{|lcc|}
\hline $3 \times 10^{18} \mathrm{~cm}^{-3}$ & $\mathrm{p}^{+}$-GaAs Contact & $200 \mathrm{~nm}$ \\
\hline $2 \times 10^{18} \mathrm{~cm}^{-3}$ & $\mathrm{p}^{+}$-InGaP Window & $50 \mathrm{~nm}$ \\
\hline $2 \times 10^{18} \mathrm{~cm}^{-3}$ & $\mathrm{p}^{+}$-GaAs Emitter & $600 \mathrm{~nm}$ \\
\hline $2 \times 10^{17} \mathrm{~cm}^{-3}$ & $\mathrm{n}$-GaAs Base & $3 \mu \mathrm{m}$ \\
\hline $2 \times 10^{18} \mathrm{~cm}^{-3}$ & $\mathrm{n}^{+}$-InGaP BSF & $30 \mathrm{~nm}$ \\
\hline $2 \times 10^{19} \mathrm{~cm}^{-3}$ & $\mathrm{n}^{+}$-GaAs Contact & $200 \mathrm{~nm}$ \\
\hline \multicolumn{3}{|c}{ Au (100 nm) } \\
\hline 3 \\
\hline \multicolumn{3}{|c}{ Kapton film $(50 \mu \mathrm{m})$} \\
\hline
\end{tabular}

(b) ELP

\begin{tabular}{|lll|}
\hline $3 \times 10^{18} \mathrm{~cm}^{-3}$ & $\mathrm{p}^{+}$-GaAs Contact & $200 \mathrm{~nm}$ \\
\hline $2 \times 10^{18} \mathrm{~cm}^{-3}$ & $\mathrm{p}^{+}$-InGaP Window & $50 \mathrm{~nm}$ \\
\hline $2 \times 10^{18} \mathrm{~cm}^{-3}$ & $\mathrm{p}^{+}$-GaAs Emitter & $600 \mathrm{~nm}$ \\
\hline $2 \times 10^{17} \mathrm{~cm}^{-3}$ & $\mathrm{n}$-GaAs Base & $3 \mu \mathrm{m}$ \\
\hline $2 \times 10^{18} \mathrm{~cm}^{-3}$ & $\mathrm{n}^{+}$-InGaP BSF & $30 \mathrm{~nm}$ \\
\hline $2 \times 10^{19} \mathrm{~cm}^{-3}$ & $\mathrm{n}^{+}$-GaAs Contact & $200 \mathrm{~nm}$ \\
\hline \multicolumn{3}{|c}{ PDMS ( 2 $\mu \mathrm{m})$} \\
\hline \multicolumn{3}{|c}{ Glass } \\
\hline
\end{tabular}

Figure 1. (Color online) Schematic structure of (a) ELA and (b) ELP SCs.

etched by dipping the sample into HF solution and the epilayer and substrate were separated. ELA was fabricated by etching the GaAs and InGaP layers through CMP. In the ELO process of the ELP SC, the AlAs sacrificial layer was etched by immersing the grown sample in HF solution to separate the epilayer and substrate, and the separated epilayer was transferred onto a PDMS/glass substrate. After that, GaAs and InGaP layers were etched as the last step of the ELA process. The detailed procedure is described in the previous study [13]. A SC device was fabricated to study the effects of defects generated during the ELO process on solar cell efficiency. In order to investigate the defects in ELP and ELA SCs, PL experiments were performed at $300 \mathrm{~K}$. A He-Ne $(633 \mathrm{~nm})$ laser was used as the excitation light source in PL and PR measurements.

\section{Results and discussion}

In a previous study, we reported the excitation- and temperaturedependent PL results of the Ref, ELA, and ELP SCs [13]. In the PL spectra at $300 \mathrm{~K}$, strong emission signals due to the band-to-band transition in GaAs were observed around $1.425 \mathrm{eV}$. The signals with multiple peaks which were not observed in the Ref SC were observed at an energy lower than the GaAs signal in the ELA and ELP SCs. Therefore, these signals are assigned to the defect states generated during the ELO process [13]. Additional peaks were produced by the interference effect of internal multi-reflection (IMR) [8]. Defect states can affect the internal electric field $\left(\boldsymbol{F}_{\text {int }}\right)$ in the space-charge region. Since the photo-generated carriers are separated by the $\boldsymbol{F}_{\text {int }}$ and move

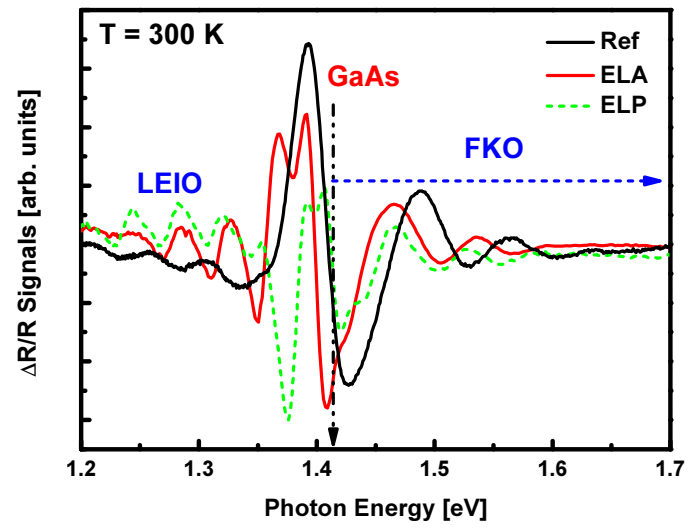

Figure 2. (Color online) PR spectra of Ref, ELA, and ELP SCs at $300 \mathrm{~K}$.

electrodes, the change of the $\boldsymbol{F}_{\text {int }}$ in the SC is very important. The presence of the effects of IMR in the PL spectra indicates that interference phenomena will also be observed in the PR spectrum.

Figure 2 shows the PR spectra of Ref, ELA, and ELP SCs at 300 $\mathrm{K}$ and the excitation intensity was $156 \mathrm{~mW} / \mathrm{cm}^{2}$. In the PR spectra of the Ref, ELA, and ELP SCs, GaAs transition signals were observed around $1.425 \mathrm{eV}$ and the Franz-Keldysh oscillations (FKOs) due to the $\boldsymbol{F}_{\text {int }}$ were also observed above the GaAs bandgap energy. Vibrating signals below the GaAs bandgap energy in the ELA and ELP SCs are low energy interference oscillations (LEIOs) $[14,15]$. In the Ref SC, the amplitude of the LEIO signal was weak and the period was shorter than those of the ELA and ELP SCs. The amplitude and period of the LEIO can be influenced by the structure, shape of the reflection, and defect state density of the thin films. The period of FKO signals was longest for the Ref SC and shortest for the ELP SC. As the FKO period is inversely proportional to the strength of the electric field [16], the $F_{i n t}$ strength is inferred to be the greatest in the Ref SC and weakest in the ELP SC. Fast Fourier transform (FFT) analysis was used to calculate the $\boldsymbol{F}_{\text {int }}$ strength more accurately [17]. The calculated $\boldsymbol{F}_{\text {int }}$ of the Ref, ELA, and ELP SCs were 122, 98.7, and $81.8 \mathrm{kV} / \mathrm{cm}$, respectively. The defect states decrease the carrier's doping concentration and thus reduce the strength of the $\boldsymbol{F}_{\text {int }}$ at the p-n junction. The $\boldsymbol{F}_{\text {int }}$ was strongest in the Ref SC, which had the lowest density of defect states. In case of the ELP SC, the thin film SC structure was completely peeled off in HF solution during the ELO process and transferred to the PDMS substrate without an external supporting force. The natural bending of the thin film during the peeling process indicated the presence of strain, which resulted in the formation of defects. However, in ELA SC, the etching process was performed with HF solution after $\mathrm{Au}$-to-Au bonding. Thus, the $\mathrm{Au}$ and polyimide substrates support the thin film so that the deformation is relatively small and fewer defects are produced. From the above results, we propose that the density of the defect states in the ELP SC was higher than that in the ELA SC.

Figure 3 shows the PR spectra of the Ref, ELA, and ELP SCs as a function of excitation intensity at $300 \mathrm{~K}$. With increasing excitation intensity, all SCs exhibited an increasing amplitude and decreasing period. The increase in amplitude was due to the increase in modulation effects as more photo-generated carriers were generated by higher excitation laser intensity. A decrease in the FKO period indicated that the strength of $\boldsymbol{F}_{\text {int }}$ was reduced. This is due to the increased photovoltage effect by the photo-generated carriers. When light is incident 

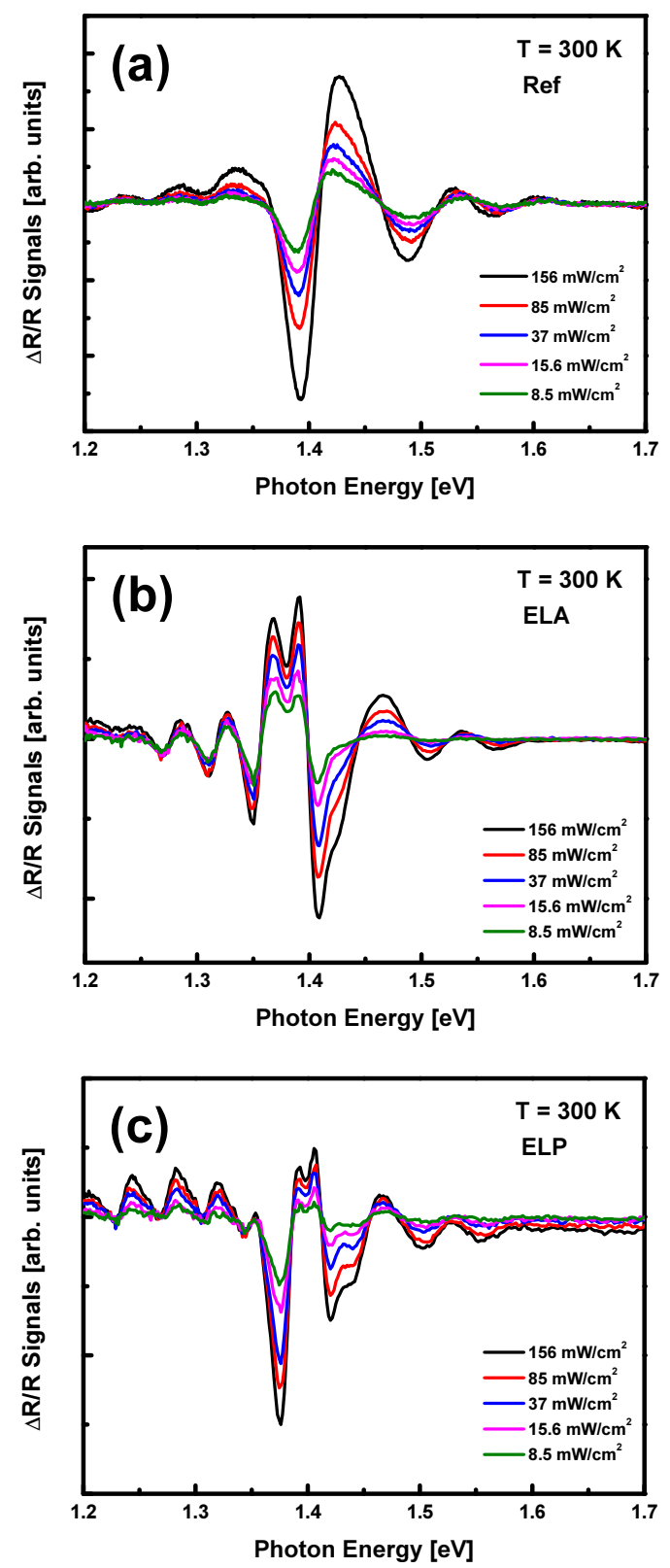

Figure 3. (Color online) PR spectra of (a) Ref, (b) ELA, and (c) ELP SCs as a function of excitation intensity at $300 \mathrm{~K}$.

onto the SC from the outside, the photo-generated carriers in the space charge region are moved by the $\boldsymbol{F}_{\text {int }}$ and accumulate on each side of the p-n junction, where they form another electric field in a direction opposite to that of $\boldsymbol{F}_{\text {int. }}$. Therefore, total $\boldsymbol{F}_{\text {int }}$ gradually decreases as excitation intensity increases.

Figure 4 shows the $\boldsymbol{F}_{\text {int }}$ (calculated with FFT) of the Ref, ELA, and ELP SCs as a function of excitation intensity at $300 \mathrm{~K}$. As the excitation intensity increases to $156 \mathrm{~mW} / \mathrm{cm}^{2}$, the $\boldsymbol{F}_{\text {int }}$ gradually decreases. If fewer photo-generated carriers are trapped by the defect states, the strength of the $\boldsymbol{F}_{\text {int }}$ will show a greater decrease. In Fig. 4, the strength of the $\boldsymbol{F}_{\text {int }}$ of the Ref SC decreased quickly, relative to that of the ELA and ELP SCs. This result is in good agreement with the presumption that there are more defects in the ELA and ELP SCs.

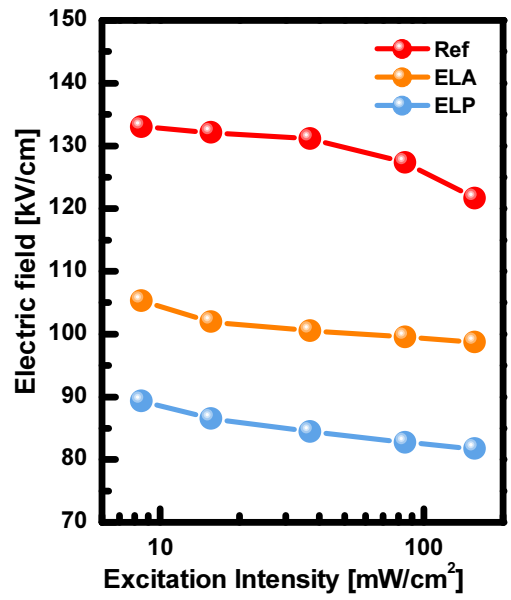

Figure 4. (Color online) Internal electric fields of Ref, ELA, and ELP SCs as functions of excitation intensity at $300 \mathrm{~K}$.

\section{Conclusions}

In this study, the defect effects generated in the GaAs SCs through the ELO process were investigated by PR spectroscopy. In the process of separating the epilayer and GaAs substrate in the ELO process, defects occurred due to the stresses that deform thin film SCs. At $156 \mathrm{~mW} / \mathrm{cm}^{2}$, the $\boldsymbol{F}_{\text {int }}$ of the Ref, ELA, and ELP SCs are 122, 98.7, and $81.8 \mathrm{kV} / \mathrm{cm}$, respectively. Lower $\boldsymbol{F}_{\text {int }}$ values are due to the relatively high defect densities of ELA and ELP SCs that are caused by stresses during the separation process. The Au-to-Au bonding process of the ELA SC suppressed much of the defect generation that occurs in ELP SC during the ELO process. To increase the efficiency of ELO SCs, more research on a technique for reducing the number of defects during ELO process will be needed.

\section{Acknowledgements}

This research was supported by the Basic Science Research Program through the National Research Foundation of Korea (NRF) funded by the Ministry of Education under grant numbers NRF-2017 R1A6A3A11028070 and NRF-2018R1D1A3B07050824. This work was supported by the Korea Institute of Energy Technology Evaluation and Planning (KETEP) and Ministry of Trade, Industry \& Energy (MOTIE) of the Republic of Korea (No. 20163030013380). This study was partially supported by the Priority Research Centers Program through the National Research Foundation of Korea (NRF) funded by the Ministry of Education (2014R1A6A1031189).

\section{References}

[1] M. A. Green, K. Emery, Y. Hishikawa, W. Warta, and E. D. Dunlop, Prog. Photovoltaics Res. Appl. 21, 1 (2013).

[2] P. Yu, C. H. Chang, C. H. Chiu, C. S. Yang, J. C. Yu, H. C. Kuo, S. H. Hsu, and Y. C. Chang, Adv. Mater. 21, 1618 (2009).

[3] J. Oh, H. C. Yuan, and H. M. Branz, Nat. Nanotechnol. 7, 743 (2012)

[4] V. Aroutiounian, S. Petrosyan, and A. Khachatryan, J. Appl. Phys. 89, 2268 (2001).

[5] Y. Cheng, M. Fukuda, V. R. Whiteside, M. C. Debnath, P. J. 
Vallely, T. D. Mishima, M. B. Santos, K. Hossain, S. Hatch, H. Y. Liu, and I. R. Sellers, Sol. Energy Mater. Sol. Cells 147, 94 (2016).

[6] Y. Yazawa, K. Tamura, S. Watahiki, T. Kitatani, J. Minemura, and T. Warabisako, Sol. Energy Mater. Sol. Cells 50, 229 (1998).

[7] Y. Kim, K. Y. Ban, C. Zhang, J. O. Kim, S. J. Lee, and C. B. Honsberg, Appl. Phys. Lett. 108, 103104 (2016).

[8] J. Maeda, Y. Sasaki, N. Dietz, K. Shibahara, S. Yokoyama, S. Miyazaki, and M. Hirose, Jpn. J. Appl. Phys. 36, 1554 (1997).

[9] Y. Sasaki, T. Katayama, T. Koishi, K. Shibahara, S. Yokoyama, S. Miyazaki, and M. Hirose, J. Electrochem. Soc. 146, 710 (1999).

[10] J. J. Schermer, G. J. Bauhuis, P. Mulder, E. J. Haverkamp, J. van Deelen, A. T. J. van Niftrik, and P. K. Larsen, Thin Solid Films 511, 645 (2006).

[11] E. Yablonovitch, D. M. Hwang, T. J. Gmitter, L. T. Florez, and J. P.
Harbison, Appl. Phys. Lett. 56, 2419 (1990).

[12] E. Yablonovitch, T. Gmitter, J. P. Harbison, and R. Bhat, Appl. Phys. Lett. 51, 2222 (1987).

[13] M. G. So, S. J. Lee, H. J. Jo, J. S. Kim, T. T. Nguyen, Y. Kim, and S. J. Lee, New Phys.: Sae Mulli. 69, 806 (2019).

[14] S. J. Lee, S. SaeidNahaiea, J. O. Kim, S. J. Lee, and J. S. Kim, Appl. Sci. Converg. Technol. 28, 46 (2019).

[15] S. Hildebrandt, M. Murtagh, R. Kuzmenko, W. Kircher, and J. Schreiber, Phys. Status Solidi A 152, 147 (1995).

[16] H. J. Jo, Y. H. Mun, J. S. Kim, and S. J. Lee, J. Korean Phys. Soc. 69, 80 (2016).

[17] M. Nowaczyk, G. Sek, J. Misiewicz, B. Sciana, D. Radziewicz, and M. Tlaczala, Thin Solid Films 380, 243 (2000). 